\title{
A homozygous MYO7A mutation associated to Usher syndrome and unilateral auditory neuropathy spectrum disorder
}

\author{
HONG XIA ${ }^{1,2^{*}}$, PENGZHI HU ${ }^{3 *}$, LAMEI YUAN $^{1}$, WEI XIONG ${ }^{4}$, HONGBO XU $^{1}$,

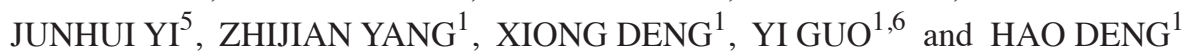 \\ ${ }^{1}$ Center for Experimental Medicine and Department of Neurology; Departments of ${ }^{2}$ Emergency and ${ }^{3}$ Radiology, \\ The Third Xiangya Hospital, Central South University, Changsha, Hunan $410013 ;{ }^{4}$ Cancer Research Institute, \\ Xiangya School of Medicine, Central South University, Changsha, Hunan 410078; ${ }^{5}$ Department of Ophthalmology, \\ The Third Xiangya Hospital, Central South University, Changsha, Hunan 410013; ${ }^{6}$ Department of Medical Information, \\ Information Security and Big Data Research Institute, Central South University, Changsha, Hunan 410083, P.R. China
}

Received May 19, 2016; Accepted March 31, 2017

DOI: $10.3892 / \mathrm{mmr} .2017 .7053$

\begin{abstract}
Usher syndrome (USH) is an autosomal recessive disorder characterized by sensorineural hearing loss, progressive visual loss and night blindness due to retinitis pigmentosa (RP), with or without vestibular dysfunction. The purpose of this study was to detect the causative gene in a consanguineous Chinese family with USH. A c.3696_3706del (p.R1232Sfs*72) variant in the myosin VIIa gene (MYO7A) was identified in the homozygous state by exome sequencing. The co-segregation of the MYO7A c.3696_3706del variant with the phenotype of deafness and progressive visual loss in the USH family was confirmed by Sanger sequencing. The variant was absent in 200 healthy controls. Therefore, the c.3696_3706del variant may disrupt the interaction between myosin VIIa and other USH1 proteins, and impair melanosome transport in retinal pigment epithelial cells. Notably, bilateral auditory brainstem responses were absent in two patients of the USH family, while distortion product otoacoustic emissions were elicited in the right ears of the two patients, consistent with clinical diagnosis of unilateral auditory neuropathy spectrum disorder. These data suggested that the homozygous c.3696_3706del variant in the MYO7A gene may be the disease-causing mutation for the disorder in this family. These findings broaden the phenotype spectrum of the MYO7A gene, and may facilitate understanding of the molecular pathogenesis of the disease, and genetic counseling for the family.
\end{abstract}

Correspondence to: Dr Hao Deng, Center for Experimental Medicine and Department of Neurology, The Third Xiangya Hospital, Central South University, 138 Tongzipo Road, Changsha, Hunan 410013, P.R. China

E-mail: hdeng008@yahoo.com

*Contributed equally

Key words: auditory neuropathy spectrum disorder, hearing loss, retinitis pigmentosa, MYO7A gene, Usher syndrome

\section{Introduction}

Usher syndrome (USH) is an autosomal recessive, clinically and genetically heterogeneous disorder. It is characterized by sensorineural hearing loss, progressive visual loss and night blindness due to retinitis pigmentosa (RP), with or without vestibular dysfunction (1-4). USH occurs in $~ 4.2-6.2$ out of 100,000 individuals $(5,6)$ and is responsible for $>50 \%$ of deaf-blindness patients (1). Hearing aids and cochlear implantation may assist hearing and oral language acquisition, while few strategies offer help to visual impairments (7).

USH is divided into three clinical subtypes based on involvement of vestibular function and the level of deafness: USH type I (USH1), type II (USH2) and type III (USH3) $(1,5)$. USH1, the most serious type, is responsible for $\sim 33-41.6 \%$ of USH patients $(5,6)$ and manifests as congenital bilateral profound sensorineural deafness with unintelligible speech, constant vestibular dysfunction since birth, and childhood onset progressive RP $(1,4)$. USH2 accounts for $46.1-47 \%$ of USH cases $(5,6)$, and patients with USH2 display non-progressive moderate to severe hearing impairments modified by hearing aids, good speech development, pubertal onset of RP and without vestibular dysfunction $(1,3)$. USH3 is characterized by post-lingual progressive moderate to severe deafness, inconstant vestibular function and the onset of RP symptoms (1).

To date, 15 loci and 11 USH genes, including myosin VIIa (MYO7A), harmonin, cadherin 23, protocadherin 15, the scaffold protein containing ankyrin repeats and SAM domain $(S A N S)$, calcium- and integrin-binding protein 2, USH type $2 \mathrm{~A}, \mathrm{G}$ protein-coupled receptor 98 , whirlin, clarin 1 and histidyl-tRNA synthetase have been associated with USH (http://hereditaryhearingloss.org). Additionally, the PDZ domain-containing 7 gene was reported as a contributor to digenic USH (8).

It is time-consuming to identify the pathogenic gene mutations in USH using regular Sanger sequencing, due to the genetic heterogeneity and large size of these USH genes (9). Exome sequencing, a cost-effectiveness strategy, was introduced to detect the causative gene mutations in USH (10). In the present study, a c.3696_3706del mutation in the MYO7A 
gene was identified in the homozygous state in a consanguineous Chinese family with USH by exome sequencing.

\section{Materials and methods}

Ethics. The present study was approved by the Institutional Review Board of the Third Xiangya Hospital, Central South University (Changsha, China) and in accordance with the Declaration of Helsinki. Written informed consent was received from all the participants or guardians.

Subjects. A four-generation Chinese Han family with USH was enlisted, and five family members took part in this study (Fig. 1A). Congenital bilateral hearing impairments, a delay in sitting and walking without assistance were observed in the two brothers (IV:3 and IV:4), and night blindness was discovered in the first decade of the proband (IV:4) and the third decade of the patient (IV:3). Neither hearing aids nor cochlear implantation was offered to the two patients during their childhood. However, their parents (III:1 and III:2) and two unaffected siblings (IV:1 and IV:2) had normal hearing, vision, speech and motor development. A total of 200 ethnically matched unrelated subjects (100 males and 100 females, aged $49.0 \pm 7.5$ years) without hearing, visual or vestibular impairments were recruited as healthy controls.

Exome sequencing and variant analysis. Genomic DNA was obtained from peripheral blood samples of all the subjects by standard method of phenol-chloroform extraction. No less than $1.5 \mu \mathrm{g}$ genomic DNA from the proband was used for exome sequencing at the Novogene Bioinformatics Institute (Beijing, China). According to the manufacturers' protocol, the genomic DNA was first sheared, and then enriched, hybridized, and captured by the Agilent SureSelect Human All Exon V5 (Agilent Technologies, Inc., Santa Clara, CA, USA), and the enriched library was sequenced using Illumina HiSeq 2000 sequencing instruments (Illumina, Inc., San Diego, CA, USA). The mean sequencing depth on the target exome was 70.80x, which covered $98.0 \%$ of the targeted exome (11).

The Burrows-Wheeler Alignment tool was applied to map the clean reads to the human reference genome (UCSC hg19, http://genome.ucsc.edu/). The Sequence Alignment/Map tools (version 1.0, http://samtools.sourceforge.net/) were used for identifying single nucleotide polymorphisms (SNPs) and insertions/deletions, and Picard (version 1.111, https://broadinstitute.github.io/picard/) was applied to mark duplicate reads. Previously reported variants detected in the SNP database version 137 (dbSNP137) with minor allele frequency $>1 \%$ and 1000 Genomes Project with a frequency of $>0.5 \%$ were screened out. Following this, variants in the exonic and splicing junction regions were retained, and synonymous variants were screened out. Sorting Intolerant from Tolerant (http://sift.jcvi. org/) and Polymorphism Phenotyping version 2 (http://genetics. bwh.harvard.edu/pph2/) were used for the functional prediction of nonsynonymous SNPs. The ANNOVAR (Annotate Variation, version 2013, August 23) software was applied to the annotation of candidate variants.

Sanger sequencing and functional prediction. The potential pathogenic variant was confirmed by Sanger sequencing using an ABI3500 sequencer (Applied Biosystems; Thermo Fisher Scientific, Inc., Waltham, MA, USA) and segregation analysis was performed (12). Two primer sequences for variants in the MYO7A gene (NM_000260.3) were designed as follows: Forward, 5'-GCTGCCCTCAAAATCCACAT-3' and reverse, 5'-CACACACGCATTTACACACG-3'. The functional prediction of the MYO7A variant was conducted using the MutationTaster online tool (http://www.mutationtaster.org/).

Clinical, audiometric and ophthalmologic evaluations. Subjects in the family had clinical, audiometric and fundus examinations conducted in the Third Xiangya Hospital. These auditory and ophthalmologic evaluations included pure tone audiometry (PTA), auditory brainstem responses (ABR), tympanometry, acoustic reflex (AR) thresholds, transient evoked otoacoustic emissions (TEOAE), distortion product otoacoustic emissions (DPOAE), magnetic resonance imaging (MRI) of the inner ear and optic nerve, the naked visual acuity, and images of the fundus with a dilated pupil. Hearing degree was distributed into healthy $(<20 \mathrm{dBHL})$, mild deafness (20-40 dBHL), moderate deafness (41-70 dBHL), severe deafness (71-95 dBHL), and profound deafness (>95 dBHL) (13).

\section{Results}

Exome sequencing. Exome sequencing of the proband generated 19,306,510 paired reads, with a mean read length of $150 \mathrm{bp}$, and $98.66 \%(19,047,947)$ of paired reads passed the quality evaluation. Approximately $99.88 \%$ clean reads were mapped to the human reference genome (14). After filtering out common variants and nonpathogenic variants, a homozygous MYO7A c.3696_3706del variant was observed in the proband, and other probable variants in the known causative genes for hearing loss or RP were excluded.

Identification of the pathogenic mutation. The MYO7A c.3696_3706del variant was confirmed in a homozygous state in the proband by Sanger sequencing (Fig. 1B). The MYO7A c.3696_3706del variant co-segregated with the phenotype of USH in the family; the homozygous c.3696_3706del variant was identified in the two affected brothers, and the heterozygous variant was detected in their unaffected father (Fig. 1C) and siblings. The MYO7A c.3696_3706del variant was predicted to result in a shift in the reading frame and a premature stop codon (p.R1232Sfs*72), the nomenclature was checked by the Mutalyzer online tool (http://www.lovd.nl/mutalyzer/). The variant was absent in two hundred healthy controls (Fig. 1D).

Clinical, audiometric and ophthalmologic results. Both patients displayed bilateral hearing loss, visual impairment and vestibular dysfunction. PTA revealed bilateral profound sensorineural deafness with thresholds $>100$ dBHL (Fig. 2A). AR, ABR (Fig. 2B) and TEOAE were absent in the two patients. A type A tympanometric curve was present in bilateral ears of the patient (IV:3) and the left ear of the proband, while a type $\mathrm{C}$ tympanometric curve was present in the right ear of the proband. No inner ear or optic nerve anomaly was detected by MRI in the two patients. DPOAE was absent in the left ears of the two patients. However, DPOAE was elicited in the right ears of the two patients (Fig. 3A). The 
A

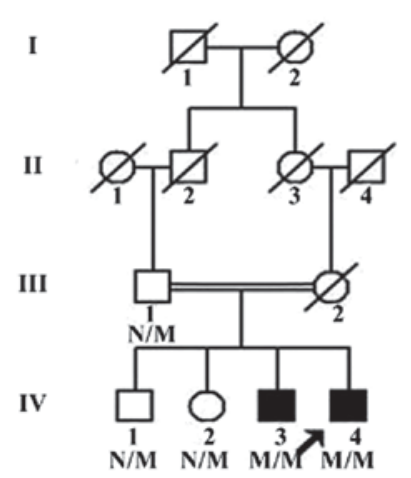

B c.3696-3706del

C G C C T G A T T A A T G G G

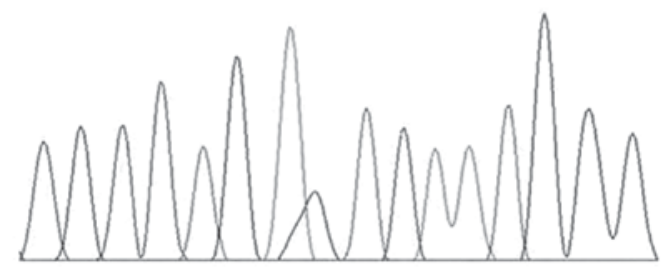

C c.3696_3706del

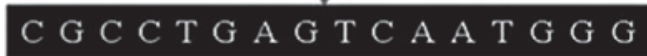

C G C C T G A G A G G A C T

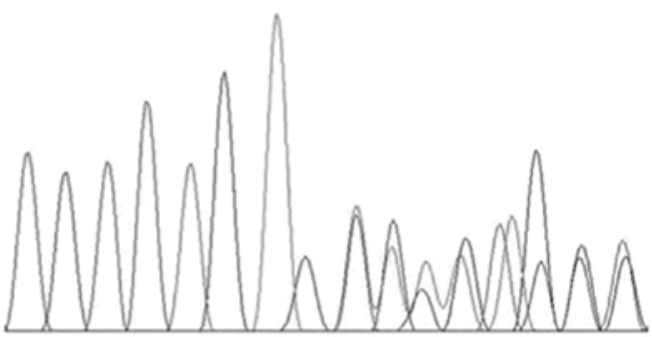

D

Figure 1. Pedigree and mutation analysis of a Chinese family with Usher syndrome. (A) Pedigree of the USH family. N, normal, M, the MYO7A c.3696_3706del variant. (B) The homozygous MYO7A c.3696_3706del variant in the affected individual (IV:4). (C) The heterozygous MYO7A c.3696_3706del variant in the unaffected individual (III:1). (D) The MYO7A gene sequence in a healthy control. MYO7A, the myosin VIIa gene.
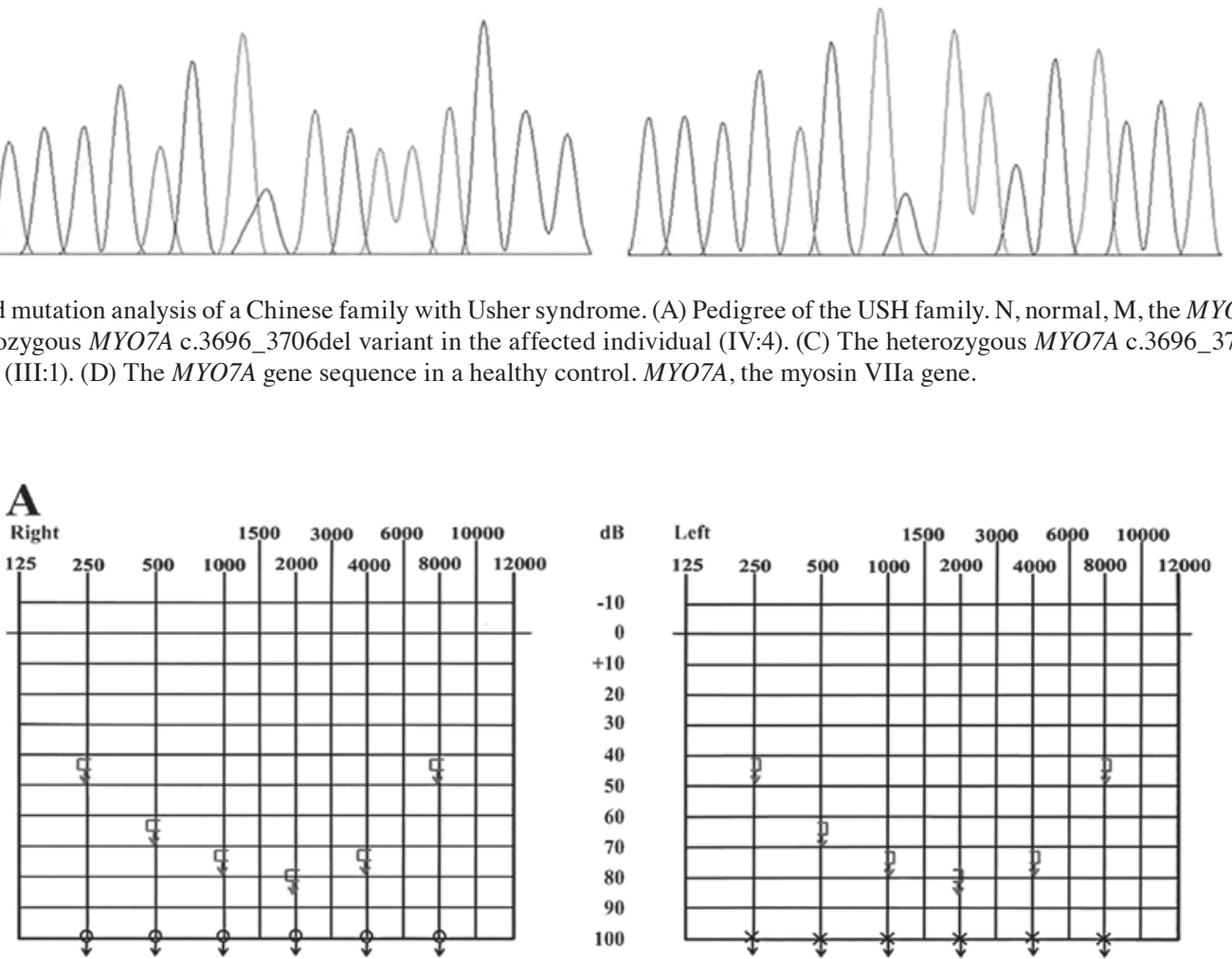

\section{B}

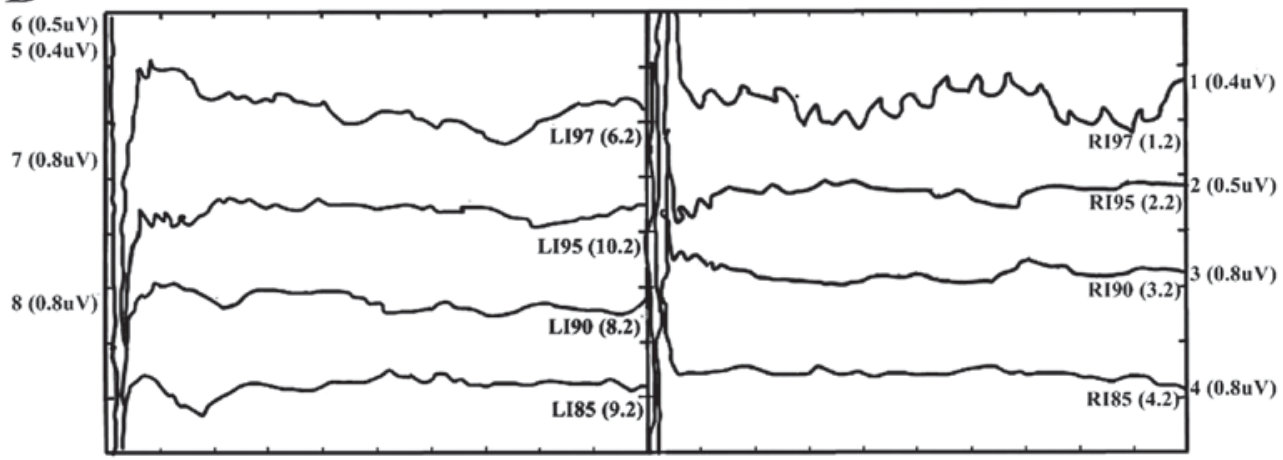

Figure 2. Audiograms and auditory brainstem responses in the proband with Usher syndrome. (A) Pure tone audiometry revealed bilateral profound sensorineural deafness. (B) Auditory brainstem responses were absent in the proband.

proband had more severe vision loss (complete blindness) and vestibular dysfunction than the patient (IV:3), whose bilateral naked visual acuity was measured as 0.1 by the $\mathrm{E}$
Standard Logarithm Visual Acuity Chart at $5 \mathrm{~m}$. Pale optic nerve, vascular attenuation and retinal bone spicule pigmentations were detected in the bilateral eyes of the two patients 
A
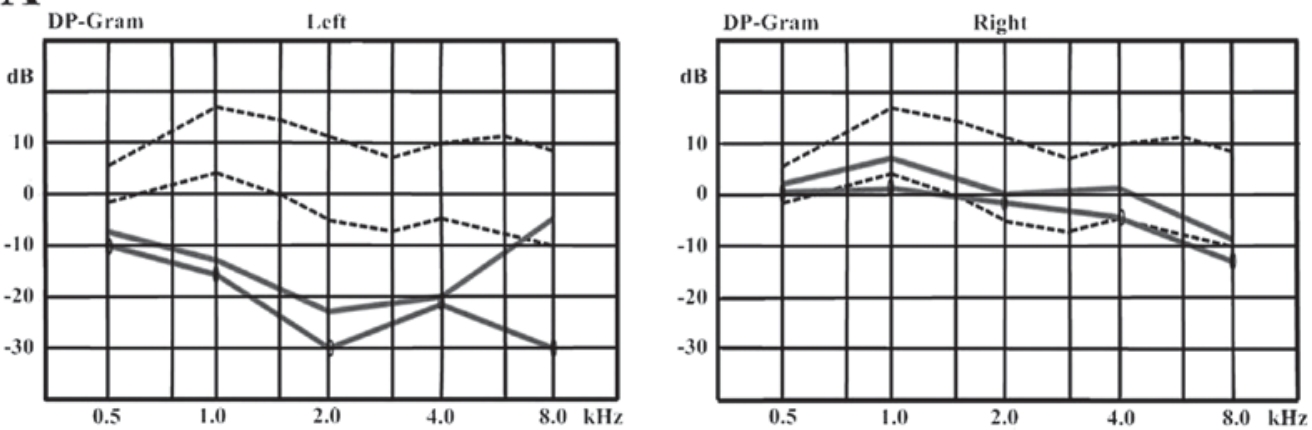

B
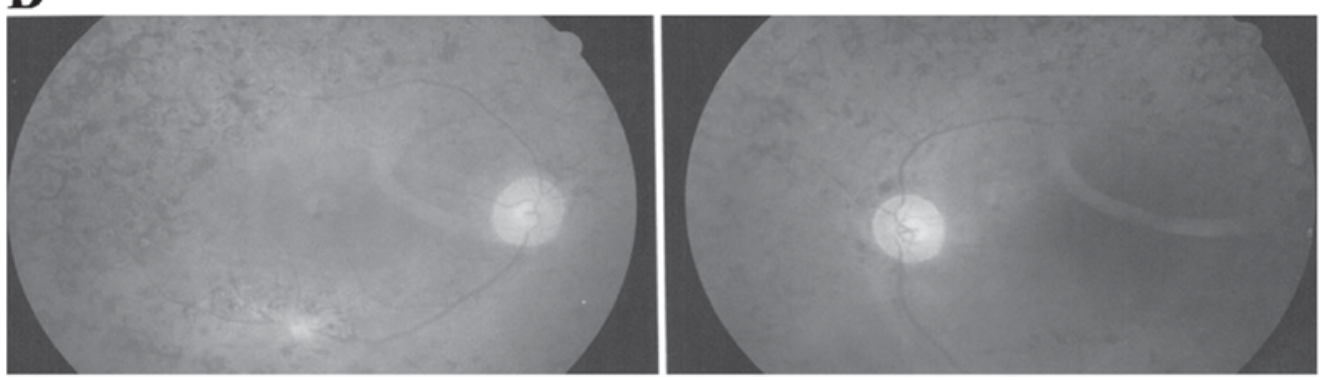

Figure 3. DPOAE and fundus images in two patients with Usher syndrome. (A) DPOAE was absent in the left ear of the proband, but was elicited in the right ear. (B) Fundus images of the patient IV:3 displayed a pale optic nerve, vascular attenuation and retinal bone spicule pigmentations. DPOAE, distortion product otoacoustic emission.

by fundus imaging (Fig. 3B). The two patients presented with bilateral profound hearing impairments, constant vestibular dysfunction and progressive retinitis pigmentosa, and were diagnosed with USH1.

\section{Discussion}

In 1992, a disease gene locus for USH type 1B (USH1B) was localized to $11 \mathrm{q} 13.5$ by linkage analysis of 27 families with USH (15). In 1995, mutations in the MYO7A gene were identified in five unrelated families with USH1B (4). Mutations in the MYO7A gene are responsible for $6.25 \%$ of Pakistani families with pre-lingual hearing loss (16). According to the Human Gene Mutation Database (http://www.hgmd.cf.ac.uk), to date $>402$ mutations in the MYO7A gene have been reported. The MYO7A p.R212C, p.R212H, p.R302H, p.R666* and p.Q821* mutations were reported to be common USH1B mutations (17-19), and p.C31* and p.R241G mutations were identified as founder mutations in the Danish and Italian populations, respectively $(20,21)$.

Of note, a few mutations in the MYO7A gene were also reported in patients with other hearing and/or visual disorders, such as autosomal recessive nonsyndromic deafness 2 (22), autosomal dominant nonsyndromic deafness 11 (23), USH2 (24) and Leber congenital amaurosis (25).

The MYO7A gene contains 49 exons, and encodes an unconventional myosin VIIa with several alternatively spliced transcripts. The long transcript is composed of 2215 amino acids, which consist of the motor domain containing a typical adenosine triphosphate binding site and an actin binding site, the regulatory domain harboring five light chain binding IQ motifs, and the tail region consisting of a coiled-coil domain, two myosin tail homology 4 (MyTH4) domains, a
Src-homology-3 (SH3) domain and two band 4.1, ezrin, radixin and moesin (FERM) domains (26-28).

The MYO7A gene is expressed in the cochlear and vestibular sensory hair cells, particularly in the actin-rich hair bundle, and in the pigment epithelium and the photoreceptor cells of the retina $(27,29)$. Myosin VIIa is implicated in sensory hair cell bundle integrity and may be essential for endocytosis at the apical end of the sensory hair cell (30). The protein also contributes to the phagocytosis of photoreceptor disk membranes by the retinal pigment epithelium, which is vital to photoreceptor cell viability (31). Defects in the morphogenesis of the inner ear sensory cell stereocilia may cause deafness and vestibular dysfunction, and pigment epithelium and photoreceptor cells may be implicated in the retinal degenerative process (27).

Both Shaker-1 mice and polka mice with homozygous Myo7a mutations displayed the phenotype of deafness and vestibular dysfunction, disorganization of the inner ear hair bundles, and defective melanosome localization in the apical retinal pigment epithelium (32-35). Defective hair bundles were also observed in zebrafish mariner mutants due to Myo7a mutations (30).

In the present study, the c.3696_3706del variant in the MYO7A gene co-segregated with the USH1 phenotype in the family, and therefore is likely to be the causative mutation. The c.3696_3706del variant (rs397516303) was previously reported in a compound heterozygous state with the $M Y O 7 A$ c.4398G $>$ A or c.4251delC variant in two Chinese patients with USH1 (36). The c.3696_3706del variant in the MYO7A gene, located in the first MyTH4 domain, was predicted to result in a shift in the reading frame and a premature stop codon (p.R1232Sfs*72), and lead to a truncated protein missing part of the first and second MyTH4 domain, the SH3 domain, and the two FERM domains in the tail region of myosin VIIa. 
Myosin VIIa may interact with other USH1 proteins, harmonin and SANS, by the C-terminal MyTH4 and FERM domains, and contribute to the shaping of hair bundles $(28,37)$. The C-terminal FERM domain of Myosin VIIa is essential for melanosome transport in the retinal pigment epithelial cells (35). The c.3696_3706del variant in the MYO7A gene may disrupt the interaction between Myosin VIIa and other USH1 proteins, and impair melanosome transport in the retinal pigment epithelial cells. Therefore, the variant may be the pathogenic mutation in the USH family, and was reported for the first time in a homozygous state.

The proband had early onset of RP and more severe phenotype than the affected brother (IV:3), potentially due to genetic modifiers or environmental factors (25). Notably, bilateral ABR was absent in both patients, while DPOAE was elicited in the right ears of the two patients, suggesting preservation of healthy cochlear outer hair cell function in their right ears. The deafness phenotypes of the two patients were consistent with clinical diagnosis of unilateral auditory neuropathy spectrum disorder (ANSD) (38). It is the first report of the association between the phenotype of ANSD and a mutation in the MYO7A gene, which may contribute to further elucidation of the molecular pathogenesis.

In conclusion, the homozygous c.3696_3706del variant in the MYO7A gene was the disease-causing mutation in this USH family. The present study indicated that exome sequencing is an effective and systematic molecular diagnostic strategy for USH, a disorder with clinical and genetic heterogeneity. These findings expand the phenotype spectrum of the MYO7A gene, and may facilitate understanding on the molecular pathogenesis and genetic counseling.

\section{Acknowledgements}

The present study was supported by grants from the National Natural Science Foundation of China (grant nos. 81271921 and 81441033), the Natural Science Foundation of Hunan Province, China (grant no. 2015JJ4088), and the Grant for the Foster Key Subject of the Third Xiangya Hospital Clinical Laboratory Diagnostics (H.D.), Zhishan Lead Project of the Third Xiangya Hospital (H.D.) and the Mittal Students' Innovative Projects of Central South University (grant nos. 15MX50 and 15MX53).

\section{References}

1. Millan JM, Aller E, Jaijo T, Blanco-Kelly F, Gimenez-Pardo A and Ayuso C: An update on the genetics of Usher syndrome. J Ophthalmol 2011: 417217, 2011.

2. Boughman JA, Vernon M and Shaver KA: Usher syndrome: Definition and estimate of prevalence from two high-risk populations. J Chronic Dis 36: 595-603, 1983.

3. Otterstedde CR, Spandau U, Blankenagel A, Kimberling WJ and Reisser C: A new clinical classification for Usher's syndrome based on a new subtype of Usher's syndrome type I. Laryngoscope 111: 84-86, 2001.

4. Weil D, Blanchard S, Kaplan J, Guilford P, Gibson F, Walsh J, Mburu P, Varela A, Levilliers J, Weston MD, et al: Defective myosin VIIA gene responsible for Usher syndrome type 1B. Nature 374: 60-61, 1995.

5. Espinós C, Millán JM, Beneyto M and Nájera C: Epidemiology of Usher syndrome in Valencia and Spain. Community Genet 1: 223-228, 1998.

6. Hope CI, Bundey S, Proops D and Fielder AR: Usher syndrome in the city of Birmingham-prevalence and clinical classification. Br J Ophthalmol 81: 46-53, 1997.
7. Bonnet $\mathrm{C}$ and El-Amraoui A: Usher syndrome (sensorineural deafness and retinitis pigmentosa): Pathogenesis, molecular diagnosis and therapeutic approaches. Curr Opin Neurol 25: 42-49, 2012.

8. Ebermann I,Phillips JB, Liebau MC, Koenekoop RK, Schermer B, Lopez I, Schäfer E, Roux AF, Dafinger C, Bernd A, et al: PDZD7 is a modifier of retinal disease and a contributor to digenic Usher syndrome. J Clin Invest 120: 1812-1823, 2010.

9. Yoshimura H, Iwasaki S, Nishio SY, Kumakawa K, Tono T, Kobayashi Y, Sato H, Nagai K, Ishikawa K, Ikezono T, et al: Massively parallel DNA sequencing facilitates diagnosis of patients with Usher syndrome type 1. PLoS One 9: e90688, 2014.

10. Reddy R, Fahiminiya S, El Zir E, Mansour A, Megarbane A, Majewski J and Slim R: Molecular genetics of the Usher syndrome in Lebanon: Identification of 11 novel protein truncating mutations by whole exome sequencing. PLoS One 9: e107326, 2014.

11. Yuan L, Guo Y, Yi J, Xiao J, Yuan J, Xiong W, Xu H, Yang Z, Zhang $\mathbf{J}$ and Deng $\mathrm{H}$ : Identification of a novel GJA3 mutation in congenital nuclear cataract. Optom Vis Sci 92: 337-342, 2015.

12. Guo Y, Yang H, Deng X, Song Z, Yang Z, Xiong W, Yuan L, $\mathrm{Xu} \mathrm{H}$, Deng S and Deng H: Genetic analysis of the S100B gene in Chinese patients with Parkinson disease. Neurosci Lett 555: 134-136, 2013.

13. Xia H, Huang X, Guo Y, Hu P, He G, Deng X, Xu H, Yang Z and Deng H: Identification of a novel MYO15A mutation in a Chinese family with autosomal recessive nonsyndromic hearing loss. PLoS One 10: e0136306, 2015.

14. Yuan L, Wu S, Xu H, Xiao J, Yang Z, Xia H, Liu A, Hu P, Lu A, Chen Y, et al: Identification of a novel PHEX mutation in a Chinese family with X-linked hypophosphatemic rickets using exome sequencing. Biol Chem 396: 27-33, 2015.

15. Kimberling WJ, Möller CG, Davenport S, Priluck IA, Beighton PH, Greenberg J, Reardon W, Weston MD, Kenyon JB, Grunkemeyer JA, et al: Linkage of Usher syndrome type I gene (USH1B) to the long arm of chromosome 11. Genomics 14: 988-994, 1992.

16. Shahzad M, Sivakumaran TA, Qaiser TA, Schultz JM, Hussain Z, Flanagan M, Bhinder MA, Kissell D, Greinwald JH Jr, Khan SN, et al: Genetic analysis through OtoSeq of Pakistani families segregating prelingual hearing loss. Otolaryngol Head Neck Surg 149: 478-487, 2013.

17. Weston MD, Kelley PM, Overbeck LD, Wagenaar M, Orten DJ, Hasson T, Chen ZY, Corey D, Mooseker M, Sumegi J, et al: Myosin VIIA mutation screening in 189 Usher syndrome type 1 patients. Am J Hum Genet 59: 1074-1083, 1996.

18. Ouyang XM, Yan D, Du LL, Hejtmancik JF, Jacobson SG, Nance WE, Li AR, Angeli S, Kaiser M, Newton V, et al: Characterization of Usher syndrome type I gene mutations in an Usher syndrome patient population. Hum Genet 116: 292-299, 2005.

19. Najera C, Beneyto M, Blanca J, Aller E, Fontcuberta A, Millán JM and Ayuso C: Mutations in myosin VIIA (MYO7A) and usherin (USH2A) in Spanish patients with Usher syndrome types I and II, respectively. Hum Mutat 20: 76-77, 2002.

20. Jaijo T, Aller E, Beney to M, Najera C, Graziano C, Turchetti D, Seri M, Ayuso C, Baiget M, Moreno F, et al: MYO7A mutation screening in Usher syndrome type I patients from diverse origins. J Med Genet 44: e71, 2007.

21. Janecke AR, Meins M, Sadeghi M, Grundmann K, Apfelstedt-Sylla E, Zrenner E, Rosenberg T and Gal A: Twelve novel myosin VIIA mutations in 34 patients with Usher syndrome type I: Confirmation of genetic heterogeneity. Hum Mutat 13: 133-140, 1999

22. Liu XZ, Walsh J, Mburu P, Kendrick-Jones J, Cope MJ, Steel KP and Brown SD: Mutations in the myosin VIIA gene cause non-syndromic recessive deafness. Nat Genet 16: 188-190, 1997.

23. Luijendijk MW, Van Wijk E, Bischoff AM, Krieger E, Huygen PL, Pennings RJ, Brunner HG, Cremers CW, Cremers FP and Kremer H: Identification and molecular modelling of a mutation in the motor head domain of myosin VIIA in a family with autosomal dominant hearing impairment (DFNA11). Hum Genet 115: 149-156, 2004.

24. Rong W, Chen X, Zhao K, Liu Y, Liu X, Ha S, Liu W, Kang X, Sheng $X$ and Zhao C: Novel and recurrent MYO7A mutations in Usher syndrome type 1 and type 2. PLoS One 9: e97808, 2014.

25. Wang X, Wang H, Cao M, Li Z, Chen X, Patenia C, Gore A, Abboud EB, Al-Rajhi AA, Lewis RA, et al: Whole-exome sequencing identifies ALMS1, IQCB1, CNGA3, and MYO7A mutations in patients with Leber congenital amaurosis. Hum Mutat 32: 1450-1459, 2011. 
26. Kelley PM, Weston MD, Chen ZY, Orten DJ, Hasson T, Overbeck LD, Pinnt J, Talmadge CB, Ing P, Mooseker MS, et al: The genomic structure of the gene defective in Usher syndrome type Ib (MYO7A). Genomics 40: 73-79, 1997.

27. Weil D, Levy G, Sahly I, Levi-Acobas F, Blanchard S, El-Amraoui A, Crozet F, Philippe H, Abitbol M and Petit C: Human myosin VIIA responsible for the Usher 1B syndrome: A predicted membrane-associated motor protein expressed in developing sensory epithelia. Proc Natl Acad Sci USA 93: 3232-3237, 1996.

28. Wu L, Pan L, Wei Z and Zhang M: Structure of MyTH4-FERM domains in myosin VIIa tail bound to cargo. Science 331: 757-760, 2011.

29. Coffin AB, Dabdoub A, Kelley MW and Popper AN: Myosin VI and VIIa distribution among inner ear epithelia in diverse fishes. Hear Res 224: 15-26, 2007.

30. Ernest S, Rauch GJ, Haffter P, Geisler R, Petit C and Nicolson T: Mariner is defective in myosin VIIA: A zebrafish model for human hereditary deafness. Hum Mol Genet 9: 2189-2196, 2000.

31. Gibbs D, Kitamoto J and Williams DS: Abnormal phagocytosis by retinal pigmented epithelium that lacks myosin VIIa, the Usher syndrome 1B protein. Proc Natl Acad Sci USA 100: 6481-6486, 2003.

32. Lefevre G, Michel V, Weil D, Lepelletier L, Bizard E, Wolfrum U, Hardelin JP and Petit C: A core cochlear phenotype in USH1 mouse mutants implicates fibrous links of the hair bundle in its cohesion, orientation and differential growth. Development 135: $1427-1437,2008$
33. Gibbs D, Cideciyan AV, Jacobson SG and Williams DS: Retinal pigment epithelium defects in humans and mice with mutations in MYO7A: Imaging melanosome-specific autofluorescence. Invest Ophthalmol Vis Sci 50: 4386-4393, 2009.

34. Steel KP: Inherited hearing defects in mice. Annu Rev Genet 29: 675-701, 1995.

35. Schwander M, Lopes V, Sczaniecka A, Gibbs D, Lillo C, Delano D, Tarantino LM, Wiltshire T, Williams DS and Müller U: A novel allele of myosin VIIa reveals a critical function for the C-terminal FERM domain for melanosome transport in retinal pigment epithelial cells. J Neurosci 29: 15810-15818, 2009.

36. Jiang L, Liang X, Li Y, Wang J, Zaneveld JE, Wang H, Xu S, Wang K, Wang B, Chen R and Sui R: Comprehensive molecular diagnosis of 67 Chinese Usher syndrome probands: High rate of ethnicity specific mutations in Chinese USH patients. Orphanet J Rare Dis 10: 110, 2015.

37. Boëda B, El-Amraoui A, Bahloul A, Goodyear R, Daviet L, Blanchard S, Perfettini I, Fath KR, Shorte S, Reiners J, et al: Myosin VIIa, harmonin and cadherin 23, three Usher I gene products that cooperate to shape the sensory hair cell bundle. Embo J 21: 6689-6699, 2002.

38. Manchaiah VK, Zhao F, Danesh AA and Duprey R: The genetic basis of auditory neuropathy spectrum disorder (ANSD). Int J Pediatr Otorhinolaryngol 75: 151-158, 2011. 